\title{
Scaling Group Transformation for MHD Boundary Layer Slip Flow of a Nanofluid over a Convectively Heated Stretching Sheet with Heat Generation
}

\author{
Md. Jashim Uddin, ${ }^{1}$ W. A. Khan, ${ }^{2}$ and A. I. Md. Ismail ${ }^{1}$ \\ ${ }^{1}$ School of Mathematical Sciences, Universiti Sains Malaysia, 11800 Penang, Malaysia \\ ${ }^{2}$ Department of Engineering Sciences, PN Engineering College, National University of \\ Sciences and Technology, Karachi 75350, Pakistan
}

Correspondence should be addressed to Md. Jashim Uddin, jashim_74@yahoo.com

Received 9 February 2012; Accepted 13 March 2012

Academic Editor: Tadeusz Kaczorek

Copyright (c) $2012 \mathrm{Md}$. Jashim Uddin et al. This is an open access article distributed under the Creative Commons Attribution License, which permits unrestricted use, distribution, and reproduction in any medium, provided the original work is properly cited.

\begin{abstract}
Steady viscous incompressible MHD laminar boundary layer slip flow of an electrically conducting nanofluid over a convectively heated permeable moving linearly stretching sheet has been investigated numerically. The effects of Brownian motion, thermophoresis, magnetic field, and heat generation/absorption are included in the nanofluid model. The similarity transformations for the governing equations are developed. The effects of the pertinent parameters, Lewis number, magnetic field, Brownian motion, heat generation, thermophoretic, momentum slip and Biot number on the flow field, temperature, skin friction factor, heat transfer rate, and nanoparticle, volume fraction rate are displayed in both graphical and tabular forms. Comparisons of analytical (for special cases) and numerical solutions with the existing results in the literature are made and is found a close agreement, that supports the validity of the present analysis and the accuracy of our numerical computations. Results for the reduced Nusselt and Sherwood numbers are provided in tabular and graphical forms for various values of the flow controlling parameters which govern the momentum, energy, and the nanoparticle volume fraction transport in the MHD boundary layer.
\end{abstract}

\section{Introduction}

Nanoparticles are made from various materials, such as oxide ceramics $\left(\mathrm{Al}_{2} \mathrm{O}_{3}, \mathrm{CuO}\right)$, nitride ceramics $(\mathrm{AlN}, \mathrm{SiN})$, carbide ceramics $(\mathrm{SiC}, \mathrm{TiC})$, metals $(\mathrm{Cu}, \mathrm{Ag}, \mathrm{Au})$, semiconductors, $\left(\mathrm{TiO}_{2}, \mathrm{SiC}\right)$, carbon nanotubes, and composite materials such as alloyed nanoparticles or nanoparticle core-polymer shell composites. According to Prodanovi et al. [1], an ultrafine nanoparticle in nanofluids is capable of flowing in porous media, and these flows can improve oil recovery; hence, nanoparticles are able to control the processes of oil recovery. 
To improve oil recovery of viscous oils, a fluid, for example water, is injected into the porous medium to displace the oil, since water viscosity is inferior to that of oil. However, increasing the injected fluid viscosity using nanofluids would drastically increase the recovery efficiency. Nanoparticles can also be used to determine changes in fluid saturation and reservoir properties during oil and gas production. A suspension of nanoparticles with base fluid to get the maximum possible thermal properties at the minimum possible concentrations (preferably $<1 \%$ by volume) by uniform dispersion and stable suspension of nanoparticles (preferably $<10 \mathrm{~nm}$ ) in host fluids is regarded as nanofluid [2,3]. According to Kandasamy et al. [4], the thermal conductivity of the ordinary heat transfer fluids is not sufficient to meet current cooling rate requirements. Nanofluids are capable of increasing the thermal conductivity and convective heat transfer performance of the base liquids. Previous researchers have pointed out that a small amount ( $<1 \%$ volume fraction) of $\mathrm{Cu}$ nanoparticles or carbon nanotubes dispersed in ethylene glycol or oil is found to enhance the poor thermal conductivity of the liquid by $40 \%$ and $150 \%$, respectively. Conventional particle-liquid suspensions require high concentrations $(>10 \%)$ of particles to achieve such enhancement. Many studies on nanofluids are being conducted by scientists and engineers due to their diverse technical and biomedical applications. Examples include (i) nanofluid collant: electronics cooling, vehicle cooling, transformer cooling, super powerful and small computers cooling, electronic devices cooling, and so on; (ii) medical applications: cancer therapy and safer surgery by cooling; (iii) process industries: materials and chemicals, detergency, food and drink, oil and gas, paper and printing, and textiles. Advances in nanoelectronics, nanophotonics, and nanomagnetics have seen the arrival of nanotechnology as a distinct discipline in its own right [5]. Ultrahighperformance cooling is necessary for many industrial technologies. However, poor thermal conductivity is a drawback in developing energy-efficient heat transfer fluids necessary for ultrahigh-performance cooling. A possible mechanism to increase the thermal conductivity of nanofluids is the Brownian motions of the nanoparticles inside the base fluids [4].

Many research papers have been published on nanofluids to understand their performance so that they can be utilized to enhance the heat transfer in various applications. A comprehensive study of convective transport in nanofluids was made by Buongiorno and $\mathrm{Hu}$ [6] and Buongiorno [7]. Kuznetsov and Nield [8] presented a similarity solution of natural convective boundary layer flow of a nanofluid past a vertical plate. They have shown that the reduced Nusselt number is a decreasing function of each of buoyancy-ratio number $N r$, a Brownian motion number $N b$, and a thermophoresis number $N t$. An analytical study on the onset of convection in a horizontal layer of a porous medium with the Brinkman model and the Darcymodel filled with a nanofluid was presented by Kuznetsov and Nield [9, 10]. In 2008, Duangthongsuk and Wongwises [11] investigated the influence of thermophysical properties of nanofluids on the convective heat transfer and potted different models used by the investigators for predicting the thermophysical properties of nanofluids. In 2009, Abu-Nada and Oztop [12] studied the inclination effect on natural convection in enclosures filled with $\mathrm{Cu}$-water nanofluid. Steady boundary layer flow and heat transfer for different types of nanofluids near a vertical plate with heat generation effects was studied by Rana and Bhargava [13]. Chamkha and Aly [14] presented a nonsimilar solution of boundary layer flow of a nanofluid near a porous vertical plate with magnetic field and heat generation/absorption effects numerically. Gorla and Chamkha [15] studied natural convection flow past a horizontal plate in a porous medium filled with a nanofluid. Magnetic nanofluids are easy to manipulate with an external magnetic field, they have been used for a variety of studies. The transpiration velocity influence on boundary layer for a non-Newtonian fluid past a vertical cone located in a porous medium filled with a nanofluid was 
investigated numerically by an efficient implicit finite-difference method by Rashad et al. [16]. According to Kandasamy et al. [4], particle transport and deposition in flowing suspensions onto surfaces is important in a broad field of applications.

The fluid flow over a surface that stretches linearly has been extensively studied because of its many practical applications in polymer processing industries, paper production, biological processes, wire drawing, metal spinning, hot rolling, and so forth. Knowledge of the heat and flow characteristics of the process is important so that the finished product meets the required quality specifications. According to Kandasamy et al. [4], a large number of problems involving heat, mass, and Newtonian and Non-Newtonian fluid flow over a stretching sheet have been investigated. These investigations involved the inclusion of the electric and magnetic fields, subject to different velocity boundary conditions such as no slip, slip, power law, or exponential variation of the stretching velocity and different thermal boundary conditions such as isothermal heat flux and constant or variable surface temperature. Crane [17] first obtained the analytical solution in case of boundary layer flow of an incompressible viscous fluid over a stretching sheet. Following him many researchers such as Liao [18], Vajravelu and Cannon [19], Raptis and Perdikis [20], Bakier [21], Abel et al. [22], and Pantokratoras [23] have studied heat and/or mass transfer over radiating/nonradiating linear/nonlinear stretching sheet. Abel et al. [22] presented numerical solutions of steady laminar MHD boundary layer flow over a vertical nonlinear stretching sheet with partial slip. The magnetohydrodynamic liquid flow and heat transfer over nonlinear permeable stretching surface in the presence of chemical reactions and partial slip were studied by Yazdi et al. [24]. Very recently, Makinde and Aziz [25] studied boundary layer flow of a nanofluid over a linearly stretching sheet taking into account the thermal convective boundary condition. By varying the magnetic field, we can affect the physical properties of these fluids.

The above literature survey reveals that all of these studies are restricted to isothermal or isoflux boundary conditions. The use of the thermal convective boundary condition in order to study Blasius flow over a flat plate was first introduced by Aziz [26]. After his pioneering work, several authors used this boundary condition to study transport phenomena. Examples include Hamad et al. [27], Yao et al. [28], Makinde and Aziz [25], Yacob et al. [29]. In all of the above studies, conventional no-slip boundary condition was used at the surface. However, fluid flows in micro/nanoscale-scale devices such as micronozzles, micropumps, microturbines, microscales heat exchangers, microreactors, microvalves, turbines, sensors, and microactuators, are important for micro- and nanoscience and the conventional no-slip boundary condition at the solid-fluid interface must be replaced with the slip condition [30-33].

Scaling group analysis is a mathematical tool to find all symmetries of the system of differential equations with auxiliary conditions and requires no prior knowledge of the equation under investigation. Symmetry groups are invariant transformations that do not alter the structural form of the investigated equation(s) [34]. In case of scaling group of transformations, the group-invariant solutions are the well-known similarity solutions of initial/ boundary value problems [35]. It is a powerful, sophisticated, and systematic method to generate similarity solutions of the governing partial differential equations. Application of scaling group analysis will reduce the number of independent variables of the governing system of partial differential equations by one and will keep the system and auxiliary conditions invariant and combine the independent variables into a single independent variable (called similarity variable) $[36,37]$. Also, the original initial and boundary conditions become two boundary conditions in the new combined variable (White and Subramanian, 2010). The main advantage of this method is that it can be successfully applied to nonlinear differential 
equations, for example, Pakdemirli and Yurusoy, [38], Boutros et al. [39], Keçebaş and Yurusoy [40], Salem [41], Puvi Arasu et al. [42], Uddin et al. [43], and Hamad et al. [27] to solve problems of fluid mechanics and heat transfer. Avramenko et al. [44] presented the symmetrical properties of the turbulent boundary-layer flows and other turbulent flows are studied utilizing the Lie group theory technique. Very recently Mukhopadhyay and Layek [45] studied the boundary layer flow and heat transfer of a fluid through a porous medium towards a stretching sheet in presence of heat generation or absorption. In the context of our study, invariant solutions are meant to be a reduction of partial differential equations to the ordinary differential equations with relevant boundary conditions. From the literature survey conducted, it seems that the combined effect of thermophoresis and Brownian motion with slip boundary condition and heat generation on MHD boundary layer flow of a nanofluid over a permeable linearly stretching sheet has not been investigated yet and this motivates our present study.

The objective of this study is to extend the work of Makinde and Aziz [25] for MHD slip flow of an electrically conducting nanofluid over a stretching sheet in the presence of heat generation/absorption. This problem is associated with many applications in the fields of metallurgy, chemical engineering, and so forth A good number of industrial processes concerning polymers involve the cooling of continuous strips or filaments by drawing them through a quiescent fluid. The final products depend on the cooling rate, which is governed by the structure of the boundary layer around the stretching surface. The governing partial differential equations have been reduced to a two-point boundary value problem in similarity variables developed by scaling group of transformations. The reduced equations have been solved numerically by an efficient Runge-Kutta-Fehlberg fourth-fifth-order numerical method under Maple 13. The effects of the embedded flow controlling parameters on the fluid velocity, temperature, skin friction factor, heat transfer rate, and the nanoparticle volume fraction rate have been demonstrated graphically and discussed.

\section{Governing Equations}

We consider a two-dimensional problem with coordinate system in which the $\bar{x}$-axis is aligned horizontally and the $\bar{y}$-axis is normal to it. A uniform transverse magnetic field of strength $B_{0}$ is applied parallel to the $\bar{y}$-axis. The induced magnetic field, the external electric field, and the electric field due to the polarization of charges are assumed to be negligible. The temperature $T$ and the nanoparticle volume fraction $C$ take constant values $T_{w}$ and $C_{w}$, respectively, at the wall and constant values, $T_{\infty}$ and $C_{\infty}$, respectively far away from the wall. The bottom of the sheet is heated by convection from a hot fluid at temperature $T_{f}$, which produces a heat transfer coefficient $h$. Assume that $T_{f}>T_{w}>T_{\infty}$. The OberbeckBoussinesq approximation is utilized and the four field equations are the conservation of mass, momentum, thermal energy, and the nanoparticles volume fraction. These equations can be written in terms of dimensional forms as [25]

$$
\begin{aligned}
\frac{\partial \bar{u}}{\partial \bar{x}}+\frac{\partial \bar{v}}{\partial \bar{y}} & =0, \\
\frac{\partial p}{\partial \bar{x}} & =\mu\left(\frac{\partial^{2} \bar{u}}{\partial x^{2}}+\frac{\partial^{2} \bar{u}}{\partial \bar{y}^{2}}\right)-\sigma B_{0}{ }^{2} \bar{u}-\rho_{f}\left(\bar{u} \frac{\partial \bar{u}}{\partial \bar{x}}+v \frac{\partial \bar{u}}{\partial \bar{y}}\right),
\end{aligned}
$$




$$
\begin{aligned}
\frac{\partial p}{\partial \bar{x}}= & \mu\left(\frac{\partial^{2} \bar{v}}{\partial \bar{x}^{2}}+\frac{\partial^{2} \bar{v}}{\partial \bar{y}^{2}}\right)-\sigma B_{0}^{2} \bar{v}-\rho_{f}\left(\bar{u} \frac{\partial \overline{\bar{w}}}{\partial x}+\bar{v} \frac{\partial \bar{v}}{\partial \bar{y}}\right) \\
\bar{u} \frac{\partial T}{\partial \bar{x}}+\bar{v} \frac{\partial T}{\partial \bar{y}}= & \alpha\left(\frac{\partial^{2} T}{\partial \bar{x}^{2}}+\frac{\partial^{2} T}{\partial \bar{y}^{2}}\right)+\tau\left\{D_{B}\left(\frac{\partial C}{\partial \bar{x}} \frac{\partial T}{\partial \bar{x}}+\frac{\partial C}{\partial \bar{y}} \frac{\partial T}{\partial \bar{y}}\right)+\frac{D_{T}}{T_{\infty}}\left[\left(\frac{\partial T}{\partial \bar{x}}\right)^{2}+\left(\frac{\partial T}{\partial \bar{y}}\right)^{2}\right]\right\} \\
& +\frac{1}{(\rho c)_{p}} Q_{0}\left(T-T_{\infty}\right) \\
\bar{u} \frac{\partial C}{\partial \bar{x}}+\bar{v} \frac{\partial C}{\partial \bar{y}}= & D_{B}\left(\frac{\partial^{2} C}{\partial \bar{x}^{2}}+\frac{\partial^{2} C}{\partial \bar{y}^{2}}\right)+\frac{D_{T}}{T_{\infty}}\left(\frac{\partial^{2} T}{\partial \bar{x}^{2}}+\frac{\partial^{2} T}{\partial \bar{y}^{2}}\right)
\end{aligned}
$$

where $\alpha=k /(\rho c)_{f}$ is the thermal diffusivity of the fluid and $\tau=(\rho c)_{p} /(\rho c)_{f}$ is the ratio of heat capacity of the nanoparticle and fluid.

The appropriate boundary conditions are [27]

$$
\begin{gathered}
\bar{u}=\bar{u}_{w}=c_{1} \bar{x}+N_{1} v \frac{\partial \bar{u}}{\partial \bar{y}^{\prime}}, \quad \bar{v}=v_{w}, \quad-\kappa \frac{\partial T}{\partial \bar{y}}=h\left(T_{f}-T\right), \quad C=C_{w} \quad \text { at } \bar{y}=0, \\
\bar{u} \longrightarrow 0, \quad T \longrightarrow T_{\infty}, \quad C \longrightarrow C_{\infty} \quad \text { as } \bar{y} \longrightarrow \infty
\end{gathered}
$$

Here, $(\bar{u}, \bar{v})$ are the velocity components along $\bar{x}$ and $\bar{y}$ axes, $v_{w}$ the suction/injection velocity, and $N_{1}$ the velocity slip factor with dimension (velocity) ${ }^{-1}$. Also $c_{1}$ is positive constant standing for the characteristic stretching intensity. $N_{1}$ is the velocity slip factor with dimension (velocity) ${ }^{-1}$. Here, $\rho_{f}$ is the density of the base fluid, $\sigma$ is the electric conductivity, $Q_{0}$ is the heat generation/absorption constant with $Q_{0}>0$ heat generation(source) and $Q_{0}<0$ heat absorption (sink), $\mu$ is the dynamic viscosity of the base fluid, $\rho_{P}$ is the density of the nanoparticles, $\left(\rho C_{P}\right)_{f}$ is the heat effective heat capacity of the fluid, $\left(\rho C_{P}\right)_{P}$ is the effective heat capacity of the nanoparticle material, $\kappa_{m}$ is the effective thermal conductivity of the porous medium, $\varepsilon$ is the porosity, $D_{B}$ is the Brownian diffusion coefficient, and $D_{T}$ signifies the thermophoretic diffusion coefficient. Performing an order of magnitude analysis of the momentum, energy, and the nanoparticle volume fraction equations and hence using the following nondimensional variables:

$$
\begin{gathered}
x=\frac{\bar{x}}{\sqrt{v / c_{1}}}, \quad y=\frac{\bar{y}}{\sqrt{v / c_{1}}}, \quad u=\frac{\bar{u}}{\sqrt{c_{1} v}}, \quad v=\frac{\bar{v}}{\sqrt{c_{1} v}}, \quad \theta=\frac{T-T_{w}}{T_{w}-T_{\infty}}=\frac{T-T_{w}}{\Delta T}, \\
\phi=\frac{C-C_{\infty}}{C_{w}-C_{\infty}}=\frac{C-C_{w}}{\Delta C}, \quad \Delta T=T_{w}-T_{\infty}, \quad \Delta C=C_{w}-C_{\infty},
\end{gathered}
$$


we have

$$
\begin{gathered}
\Delta_{1} \equiv \frac{\partial \psi}{\partial y} \frac{\partial^{2} \psi}{\partial x \partial y}-\frac{\partial \psi}{\partial x} \frac{\partial^{2} \psi}{\partial y^{2}}-\frac{\partial^{3} \psi}{\partial y^{3}}+M \frac{\partial \psi}{\partial y}=0, \\
\Delta_{2} \equiv \frac{\partial \psi}{\partial y} \frac{\partial \theta}{\partial x}-\frac{\partial \psi}{\partial x} \frac{\partial \theta}{\partial y}-\frac{1}{\operatorname{Pr}} \frac{\partial^{2} \theta}{\partial y^{2}}-N b \frac{\partial \theta}{\partial y} \frac{\partial \phi}{\partial y}-N t\left(\frac{\partial \theta}{\partial y}\right)^{2}-Q \theta=0, \\
\Delta_{3} \equiv L e\left[\frac{\partial \psi}{\partial y} \frac{\partial \phi}{\partial x}-\frac{\partial \psi}{\partial x} \frac{\partial \phi}{\partial y}\right]-\frac{\partial^{2} \phi}{\partial y^{2}}-\frac{N t}{N b} \frac{\partial^{2} \theta}{\partial y^{2}}=0 .
\end{gathered}
$$

The boundary conditions in (2.2) become

$$
\begin{gathered}
\frac{\partial \psi}{\partial y}=x+a \frac{\partial^{2} \psi}{\partial y^{2}}, \quad \frac{\partial \psi}{\partial x}=f w, \quad \frac{\partial \theta}{\partial y}=-B i(1-\theta), \quad \phi=1 \quad \text { at } y=0, \\
\frac{\partial \psi}{\partial y} \longrightarrow 0, \quad \theta \longrightarrow 0, \quad \phi \longrightarrow 0 \quad \text { as } y \longrightarrow \infty
\end{gathered}
$$

where $\psi$ is the stream function defined by $u=\partial \psi / \partial y$ and $v=-\partial \psi / \partial x$ satisfies the continuity equation automatically. The nine parameters involved in (2.4)-(2.7) are defined as

$$
\begin{gathered}
M=\frac{\sigma B_{0}^{2}}{\rho_{f} c_{1}}, \quad Q=\frac{Q_{0}}{(\rho c)_{p} c_{1}}, \quad \operatorname{Pr}=\frac{\mu c_{p}}{\kappa}, \\
N t=\frac{\tau D_{B} \Delta T}{v T_{\infty}}, \quad N b=\frac{\tau D_{B} \Delta C}{\mathcal{v}}, \quad L e=\frac{v}{D^{\prime}} \\
f w=\frac{v_{w}}{\sqrt{c_{1} \mathcal{v}}}, \quad B i=\frac{h \sqrt{\mathcal{v}}}{\kappa c_{1}}, \quad a=\sqrt{c \mathcal{V}} N_{1} .
\end{gathered}
$$

In (2.8), $Q, M, \operatorname{Pr}, N t, N b, L e, a, f w$, and $B i$ denote the heat generation parameter, the magnetic field parameter, the Prandtl number, the thermophoresis parameter, the Brownian motion parameter, the Lewis number, the velocity slip parameter, the suction/injection parameter, and the Biot number, respectively (see [48]).

\section{Application of Scaling Group of Transformations}

A one-parameter scaling group of transformations that is a simplified form of the Lie group transformation is selected as $[49,50]$

$$
\Gamma: x^{*}=x e^{\varepsilon \alpha_{1}}, \quad y^{*}=y e^{\varepsilon \alpha_{2}}, \quad \psi^{*}=\psi e^{\varepsilon \alpha_{3}}, \quad \theta^{*}=\theta e^{\varepsilon \alpha_{4}}, \quad \phi^{*}=\phi e^{\varepsilon \alpha_{5}} .
$$


Here, $\varepsilon(\neq 0)$ is the parameter of the group and $\alpha^{\prime}$ s are arbitrary real numbers whose interrelationship will be determined by our analysis. The transformation (3.1) is treated as a point transformation that transforms the coordinates $(x, y, \psi, \theta, \phi)$ to $\left(x^{*}, y^{*}, \psi^{*}, \theta^{*}, \phi^{*}\right)$. We now investigate the relationship among the exponents $\alpha^{\prime}$ s such that

$$
\begin{aligned}
& \Delta_{j}\left(x^{*}, y^{*}, u^{*}, v^{*}, \ldots, \frac{\partial^{3} \psi^{*}}{\partial y^{* 3}}\right) \\
& \quad=H_{j}\left(x, y, u, v, \ldots, \frac{\partial^{3} \psi}{\partial y^{3}} ; a\right) \Delta_{j}\left(x, y, \ldots, \frac{\partial^{3} \psi}{\partial y^{3}}\right) \quad(j=1,2,3)
\end{aligned}
$$

since this is the requirement that the differential forms $\Delta_{1}, \Delta_{2}$, and $\Delta_{3}$ are conformally invariant under the transformation group $\Gamma$ [51]. Substituting the transformations in (3.1) into (2.4)-(2.6) leads to (see [52])

$$
\begin{aligned}
\Delta_{1} \equiv & \frac{\partial \psi^{*}}{\partial y^{*}} \frac{\partial^{2} \psi^{*}}{\partial y^{*} \partial x^{*}}-\frac{\partial \psi^{*}}{\partial x^{*}} \frac{\partial^{2} \psi^{*}}{\partial y^{* 2}}-\frac{\partial^{3} \psi^{*}}{\partial y^{* 3}}+M \frac{\partial \psi^{*}}{\partial x^{*}} \\
= & e^{\varepsilon\left(2 \alpha_{3}-2 \alpha_{2}-\alpha_{1}\right)}\left[\frac{\partial \psi}{\partial y} \frac{\partial^{2} \psi}{\partial y \partial x}-\frac{\partial \psi}{\partial x} \frac{\partial^{2} \psi}{\partial y^{2}}\right]-e^{\varepsilon\left(\alpha_{3}-3 \alpha_{2}\right)} \frac{\partial^{3} \psi}{\partial y^{3}}+M e^{\varepsilon\left(\alpha_{3}-\alpha_{2}\right)} \frac{\partial \psi}{\partial y} \\
\Delta_{2} \equiv & \frac{\partial \psi^{*}}{\partial y^{*}} \frac{\partial \theta^{*}}{\partial x^{*}}-\frac{\partial \psi^{*}}{\partial x^{*}} \frac{\partial \theta^{*}}{\partial y^{*}}-\frac{1}{\operatorname{Pr}} \frac{\partial^{2} \theta^{*}}{\partial y^{* 2}}-N b \frac{\partial \theta^{*}}{\partial y^{*}} \frac{\partial \phi^{*}}{\partial y^{*}}-N t\left(\frac{\partial \theta^{*}}{\partial y^{*}}\right)^{2}-Q \theta^{*} \\
= & e^{\varepsilon\left(\alpha_{3}+\alpha_{4}-\alpha_{1}-\alpha_{2}\right)}\left[\frac{\partial \psi}{\partial y} \frac{\partial \theta}{\partial x}-\frac{\partial \psi}{\partial x} \frac{\partial \theta}{\partial y}\right]-e^{\varepsilon\left(\alpha_{4}-2 \alpha_{2}\right)} \frac{1}{\operatorname{Pr}} \frac{\partial^{2} \theta}{\partial y^{2}} \\
& -e^{\varepsilon\left(\alpha_{4}+\alpha_{5}-2 \alpha_{2}\right)} N b \frac{\partial \theta}{\partial y} \frac{\partial \phi}{\partial y}-e^{\varepsilon\left(2 \alpha_{4}-2 \alpha_{2}\right)} N t\left(\frac{\partial \theta}{\partial y}\right)^{2}-Q e^{\varepsilon \alpha_{4}} \theta^{*}, \\
\Delta_{3} \equiv & L e\left[\frac{\partial \psi^{*}}{\partial y^{*}} \frac{\partial \phi^{*}}{\partial x^{*}}-\frac{\partial \psi^{*}}{\partial x^{*}} \frac{\partial \phi^{*}}{\partial y^{*}}\right]-\frac{\partial^{2} \phi^{*}}{\partial y^{* 2}}-\frac{N t}{N b} \frac{\partial^{2} \theta^{*}}{\partial y^{* 2}} \\
= & -e^{\varepsilon\left(\alpha_{5}-2 \alpha_{2}\right)} \frac{\partial^{2} \phi}{\partial y^{2}}+e^{\varepsilon\left(\alpha_{3}+\alpha_{5}-\alpha_{1}-\alpha_{2}\right)} L e\left[\frac{\partial \psi}{\partial y} \frac{\partial \phi}{\partial x}-\frac{\partial \psi}{\partial x} \frac{\partial \phi}{\partial y}\right]-e^{\varepsilon\left(\alpha_{4}-2 \alpha_{2}\right)} \frac{N t}{N b} \frac{\partial^{2} \theta}{\partial y^{2}} .
\end{aligned}
$$

The system will remain invariant under the group transformation $\Gamma$, and we would have the following relations among the exponents:

$$
\begin{gathered}
2 \alpha_{3}-2 \alpha_{2}-\alpha_{1}=\alpha_{3}-3 \alpha_{2}=\alpha_{3}-\alpha_{2} \\
\alpha_{3}+\alpha_{4}-\alpha_{1}-\alpha_{2}=\alpha_{4}-2 \alpha_{2}=\alpha_{4}+\alpha_{5}-2 \alpha_{2}=2 \alpha_{4}-2 \alpha_{2} \\
\alpha_{3}+\alpha_{5}-\alpha_{1}-\alpha_{2}=\alpha_{5}-2 \alpha_{2}=\alpha_{4}-2 \alpha_{2} .
\end{gathered}
$$

The boundary conditions will be invariant under $\Gamma$ if the following equations hold:

$$
\alpha_{3}-\alpha_{2}=\alpha_{1}=\alpha_{3}-2 \alpha_{2}, \quad \alpha_{3}-\alpha_{1}=0, \quad \alpha_{4}-\alpha_{2}=0=\alpha_{4}, \quad \alpha_{5}=0 .
$$


Solving (3.4) and (3.5) yields

$$
\alpha_{2}=\alpha_{4}=\alpha_{5}=0, \quad \alpha_{1}=\alpha_{3} .
$$

\subsection{Absolute Invariants}

The set of transformations $\Gamma$ reduces to

$$
x^{*}=x e^{\varepsilon \alpha_{1}}, \quad y^{*}=y, \quad \psi^{*}=\psi e^{\varepsilon \alpha_{1}}, \quad \theta^{*}=\theta, \quad \phi^{*}=\phi .
$$

The generator corresponding to the one-parameter infinitesimal Lie group of point transformations (3.7) is

$$
X=x \frac{\partial}{\partial x}+\psi \frac{\partial}{\partial \psi}
$$

The invariant $g(x, y, \psi)$ corresponding to $X$ is obtained by solving the differential equation

$$
X=x \frac{\partial g}{\partial x}+\psi \frac{\partial g}{\partial \psi}
$$

The auxiliary equation is $d x / x=d \psi / \psi$, which gives

$$
\frac{\Psi}{x}=\text { constant }=f(\eta)(\text { say }) \quad \text { with } \eta=y, \quad \theta=(\eta), \quad \phi=\phi(\eta) .
$$

\subsection{Governing Similarity Equations}

Substitution of (3.10) into (2.4)-(2.6) leads to the following similarity equations:

$$
\begin{gathered}
f^{\prime \prime \prime}+f f^{\prime \prime}-f^{\prime 2}-M f^{\prime}=0, \\
\theta^{\prime \prime}+\operatorname{Pr}\left[f \theta^{\prime}+Q \theta+N b \theta^{\prime} \phi^{\prime}+N t \theta^{\prime 2}\right]=0, \\
\phi^{\prime \prime}+\operatorname{Lef} \phi^{\prime}+\frac{N t}{N b} \theta^{\prime \prime}=0
\end{gathered}
$$

subject to the boundary conditions

$$
\begin{gathered}
f(0)=f w, \quad f^{\prime}(0)=1+a f^{\prime \prime}(0), \quad \theta^{\prime}(0)=-B i[1-\theta(0)], \\
\phi(0)=1, \quad f^{\prime}(\infty)=\theta(\infty)=\phi(\infty)=0,
\end{gathered}
$$


where primes denote differentiation with respect to $\eta$. Here, $f w>0$ corresponds to suction and $f w<0$ corresponds to injection. Following Makinde and Aziz [25], the physical quantities: the skin friction factor, the reduced Nusselt number, and the reduced Sherwood number, are directly proportional to $-f^{\prime \prime}(0),-\theta^{\prime}(0)$, and $-\phi^{\prime}(0)$ respectively.

\section{Comparisons}

It is worth mentioning that in case of purely hydromagnetic boundary layer $(M=0)$, noslip boundary condition $(a=0)$, for impermeable sheet $(f w=0)$ and in the absence of heat generation $(Q=0)$, the problem under consideration reduces to the problem that has been recently investigated by Makinde and Aziz [25]. It is also worth mentioning that in case of purely hydromagnetic boundary layer $(M=0)$, no slip boundary conditions $(a=0)$, for impermeable sheet $(f w=0)$, in the absence of heat generation $(Q=0)$ and for isothermal sheet $(B i \rightarrow \infty)$, the problem under consideration reduces to the problem that has been investigated by Khan and Pop [47].

\section{Solutions of Similarity Equations}

\subsection{Analytical Solutions}

Following Hamad et al. [27], we assume the solution of (3.11) is in the following form:

$$
f=A+B \exp (-m \eta)
$$

where $A, B$, and $m$ are parameters that will be determined so that they satisfy (3.11) as well as the boundary condition on $f$ in (3.14). Applying the boundary conditions in (3.14), it can be found that the final form of the solution of the momentum (3.11) is

$$
f(\eta)=f w+\frac{1}{m(1+a m)}[1-\exp (-m \eta)]
$$

with $m$ given by

$$
a m^{3}+(1-a f w) m^{2}-m(f w+a M)-1-M=0 .
$$

In case of no-slip boundary condition for which $a=0$, we have that the solution is

$$
f(\eta)=f w+\frac{1}{m}[1-\exp (-m \eta)]
$$

where $m=\left|f w+\sqrt{f w^{2}+4(1+M)}\right| / 2$. Hence, the flow parameter $f^{\prime \prime}(0)$ is obtained as

$$
f^{\prime \prime}(0)=-\frac{f w+\sqrt{f w^{2}+4(1+M)}}{2} .
$$


Note that for no-slip boundary condition and for impermeable plate in case of purely hydrodynamic case $(M=0)$, the solution of (3.11) becomes

$$
f(\eta)=1-e^{-\eta}
$$

Note that this solution was first found by Crane [17].

\subsection{Numerical Solution}

The set of nonlinear ordinary differential equations (3.11)-(3.13) subject to the boundary conditions in (3.14) have been solved numerically using Runge-Kutta-Fehlberg fourth-fifthorder numerical method under Maple 13 for various values of the flow controlling parameters. The accuracy of the method has been tested in various convective heat transfer problems. As a further confirmation, very recently the method was used by Aziz et al. [50] and Khan and Aziz [47] and found to reproduce their flawless results. The asymptotic boundary conditions given in (3.14) were replaced by a finite value of 10 for similarity variable $\eta_{\max }$ as follows:

$$
\eta_{\max }=10, \quad f^{\prime}(10)=\theta(10)=\phi(10)=0 .
$$

The choice of $\eta_{\max }=10$ ensured that all numerical solutions obey the far-field asymptotic values correctly. In 2009, Pantokratoras [53] noticed that the erroneous result was found by many researchers in the field of convective heat and mass transfer because of taking small far-field asymptotic value of $\eta$ during their numerical computation.

\section{Results and Discussion}

Numerical results are plotted in Figures 1 to 8 to exhibit the influences of the various flow controlling parameters on the dimensionless velocity, temperature, skin friction factor, heat transfer rate, and nanoparticle volume fraction rate. To verify the accuracy of our numerical scheme, a comparison of the computed skin friction factor is made to that of Hayat et al. [46] in Table 1 and a close agreement is found. Further, comparisons of the values of the reduced Nusselt number $-\theta^{\prime}(0)$ and the reduced Sherwood number $-\phi^{\prime}(0)$ with Khan and Pop [47] and Makinde and Aziz [25] for different values of the flow controlling parameters are shown in Tables 2, 3, 4, and 5, respectively. Comparisons show a good agreement for each value of the relevant parameters. Therefore, we are confident that the present results are very accurate.

\subsection{Velocity Profiles}

Effects of the magnetic field and suction/injection on the dimensionless velocity are shown in Figure 1. As expected, the suction reduces the velocity at the surface (Figure 1(a)) whilst injection increases the velocity (Figure 1(b)) both for the hydrodynamic $(M=0)$ and magnetohydrodynamics $(M \neq 0)$ boundary layer. It is also noticed that magnetic field reduces the velocity boundary layer thickness for both the suction and injection cases. Figure 2 illustrates the effects of velocity slip and suction/injection parameters on the dimensionless velocity. It is found from Figure 2 that the velocity slip suppresses the velocity for both the suction and injection cases. Again, the suction reduces the velocity at the surface for both 


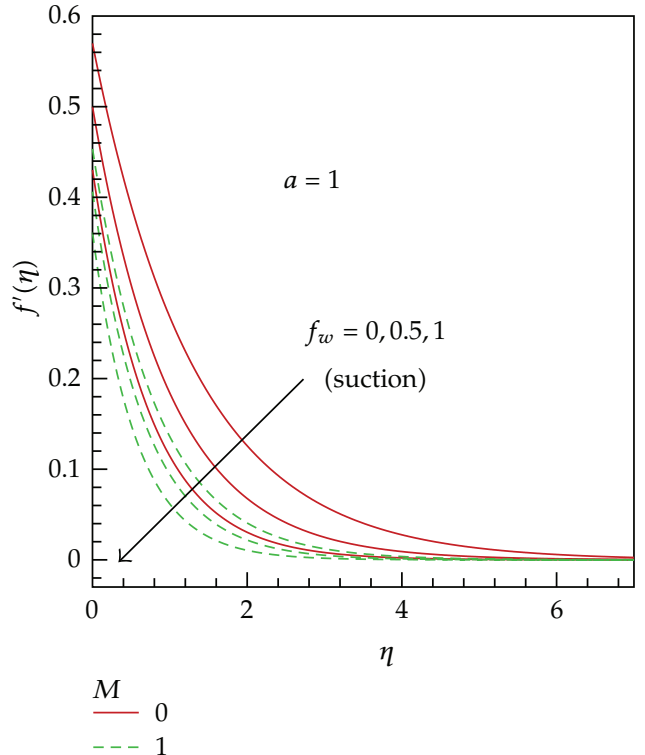

(a)

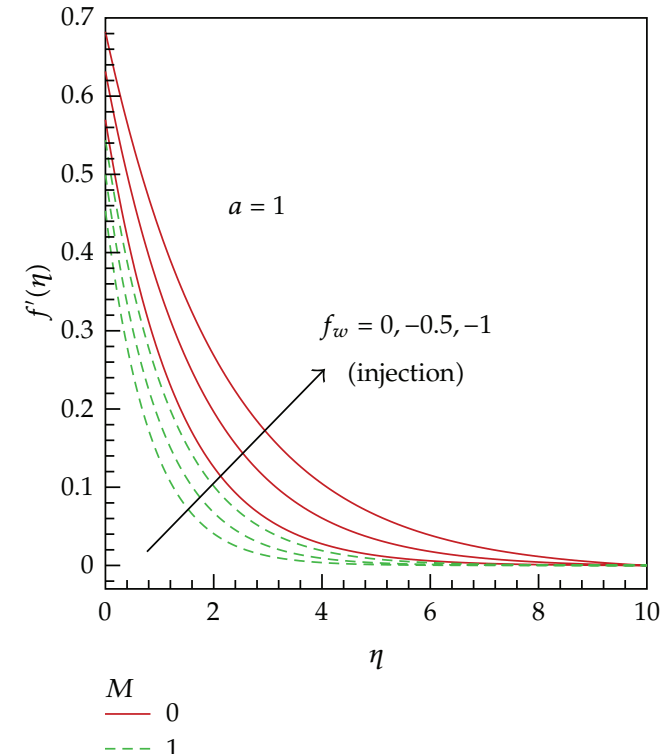

(b)

Figure 1: Effect of magnetic parameter on dimensionless velocity for (a) suction and (b) injection.

Table 1: Comparisons of dimensionless skin friction coefficient $-f^{\prime \prime}(0)$ for various values of slip parameter $a$ when $N b=N t=0, B i \rightarrow \infty, f w=0=M=Q=0$.

\begin{tabular}{lccc}
\hline$a$ & Hayat et al. [46] & Present (numerical) & Present (analytical) \\
\hline 0.0 & 1.000000 & 1.00000000 & 1.00000000 \\
0.1 & 0.872082 & 0.87208247 & 0.95540136 \\
0.2 & 0.776377 & 0.77637707 & 0.77637707 \\
0.5 & 0.591195 & 0.59119548 & 0.59119548 \\
1.0 & 0.430162 & 0.43015970 & 0.43015971 \\
2.0 & 0.283981 & 0.28397959 & 0.28397959 \\
5.0 & 0.144841 & 0.14484019 & 0.14484019 \\
10.0 & 0.081249 & 0.08124198 & 0.08124198 \\
20.0 & 0.043782 & 0.04378834 & 0.04378832 \\
50.0 & 0.018634 & 0.01859623 & 0.01859617 \\
100.0 & 0.009581 & 0.00954997 & 0.00954987 \\
\hline
\end{tabular}

Table 2: Comparison of the dimensionless heat transfer rate $-\theta^{\prime}(0)$ with previous published works for $N b=$ $N t=f w=a=M=Q=0, B i=1000, L e=1$.

\begin{tabular}{lccc}
\hline Pr & Khan and Pop [47] & Makinde and Aziz [25] & Present results \\
\hline 0.70 & 0.4539 & 0.4539 & 0.4537 \\
2.00 & 0.9113 & 0.9114 & 0.9105 \\
7.00 & 1.8954 & 1.18905 & 1.8918 \\
20.00 & 3.3539 & 3.3539 & 3.3539 \\
70.00 & 6.4621 & 6.4621 & 6.4622 \\
\hline
\end{tabular}


Table 3: Comparisons of results for reduced Nusselt number $-\theta^{\prime}(0)$ and reduced Sherwood number $-\phi^{\prime}(0)$ with $L e=\operatorname{Pr}=10, B i \rightarrow \infty$.

\begin{tabular}{llllllll}
\hline$N b$ & $N t$ & Nur [47] & Shr [47] & Nur [25] & Shr [25] & $\begin{array}{l}\text { Nur } \\
\text { (present) }\end{array}$ & $\begin{array}{l}\text { Shr } \\
\text { (present) }\end{array}$ \\
\hline 0.1 & 0.1 & 0.9524 & 2.1294 & 0.9524 & 2.1294 & 0.95238 & 2.12939 \\
0.2 & 0.1 & 0.5056 & 2.3819 & 0.5056 & 2.3819 & 0.50558 & 2.38187 \\
0.3 & 0.1 & 0.2522 & 2.4100 & 0.2522 & 2.4100 & 0.25216 & 2.41002 \\
0.4 & 0.1 & 0.1194 & 2.3997 & 0.1194 & 2.3997 & 0.11946 & 2.39965 \\
0.5 & 0.1 & 0.0543 & 2.3836 & 0.0543 & 2.3836 & 0.05425 & 2.38357 \\
0.1 & 0.2 & 0.6932 & 2.2740 & 0.6932 & 2.2740 & 0.69317 & 2.27401 \\
0.1 & 0.3 & 0.5201 & 2.5286 & 0.5201 & 2.5286 & 0.52008 & 2.52863 \\
0.1 & 0.4 & 0.4026 & 2.7952 & 0.4026 & 2.7952 & 0.40258 & 2.79515 \\
0.1 & 0.5 & 0.3211 & 3.03512 & 0.3211 & 3.03512 & 0.32105 & 3.03512 \\
\hline
\end{tabular}

Table 4: Comparisons of results for reduced Nusselt number $-\theta^{\prime}(0)$ with $L e=\operatorname{Pr}=10, B i=0.1$.

\begin{tabular}{ccccccccc}
\hline & \multicolumn{3}{c}{ Nur [25] } & \multicolumn{5}{c}{ Nur (present) } \\
$N t$ & $N b=0.1$ & $N b=0.2$ & $N b=0.3$ & $N b=0.4$ & $N b=0.1$ & $N b=0.2$ & $N b=0.3$ & $N b=0.4$ \\
\hline 0.1 & 0.0929 & 0.0873 & 0.0769 & 0.0597 & 0.09291 & 0.08733 & 0.07688 & 0.05966 \\
0.2 & 0.0927 & 0.0868 & 0.0751 & 0.0553 & 0.09273 & 0.08676 & 0.07508 & 0.05535 \\
0.3 & 0.0925 & 0.0861 & 0.0729 & 0.0503 & 0.09255 & 0.08612 & 0.07292 & 0.05027 \\
0.4 & 0.0923 & 0.0854 & 0.0703 & 0.0445 & 0.09234 & 0.08539 & 0.07027 & 0.04455 \\
0.5 & 0.0921 & 0.0845 & 0.0700 & 0.0386 & 0.09213 & 0.08454 & 0.06697 & 0.03860 \\
\hline
\end{tabular}

conventional no-slip boundary condition and Maxwell velocity slip boundary condition. Opposite behavior is observed for both slip and no-slip boundary conditions in case of injection.

\subsection{Temperature Profiles}

The variation of dimensionless temperature with Prandtl number and suction/injection parameters is depicted in Figure 3. The dimensionless temperature and corresponding thermal boundary layer decrease for suction for all values of Prandtl number. Reverse phenomena is noticed in case of injection. The dimensionless temperature decreases with an increase in Prandtl number for both suction and injection. Impact of the velocity slip and nanofluid parameters, namely, thermophoresis and Brownian motion, on the dimensionless temperature is demonstrated in Figure 4. The effect of both nanofluid parameters is to increase the dimensionless temperature with and without slip boundary condition (Figures 4(a) and 4(b)). Comparison of Figures 4(a) and 4(b) reveals that slip velocity increases the dimensionless temperature and corresponding thermal boundary layer thickness. Figure 5 illustrate the effects of Biot number and heat generation parameter on the dimensionless temperature. Increasing Biot number leads to an increase in the dimensionless temperature for both the impermeable (Figure 5(a)) and permeable (Figure 5(b)) plates and in both the presence and absence of the heat generation. The generation of heat increases the dimensionless temperature and thermal boundary layer in both cases with/without suction. This increase in the dimensionless temperature produces an increase in the velocity and temperature due 
Table 5: Comparisons of results for reduced Sherwood number $-\phi^{\prime}(0)$ with $L e=\operatorname{Pr}=10, B i=0.1$.

\begin{tabular}{ccccccccc}
\hline \multicolumn{3}{c}{ Shr [25] } & \multicolumn{5}{c}{ Shr (present) } \\
$N t$ & $N b=0.1$ & $N b=0.2$ & $N b=0.3$ & $N b=0.4$ & $N b=0.1$ & $N b=0.2$ & $N b=0.3$ & $N b=0.4$ \\
\hline 0.1 & 2.2774 & 2.3109 & 2.3299 & 2.3458 & 2.27742 & 2.31094 & 2.32994 & 2.34583 \\
0.2 & 2.2490 & 2.3168 & 2.3569 & 2.3903 & 2.24896 & 2.31682 & 2.35692 & 2.39025 \\
0.3 & 2.2228 & 2.3261 & 2.3900 & 2.4411 & 2.22281 & 2.32606 & 2.38997 & 2.44114 \\
0.4 & 2.1992 & 2.3392 & 2.4303 & 2.4967 & 2.19919 & 2.33924 & 2.43031 & 2.49667 \\
0.5 & 2.1783 & 2.3570 & 2.4792 & 2.5529 & 2.17835 & 2.35704 & 2.47923 & 2.55287 \\
\hline
\end{tabular}

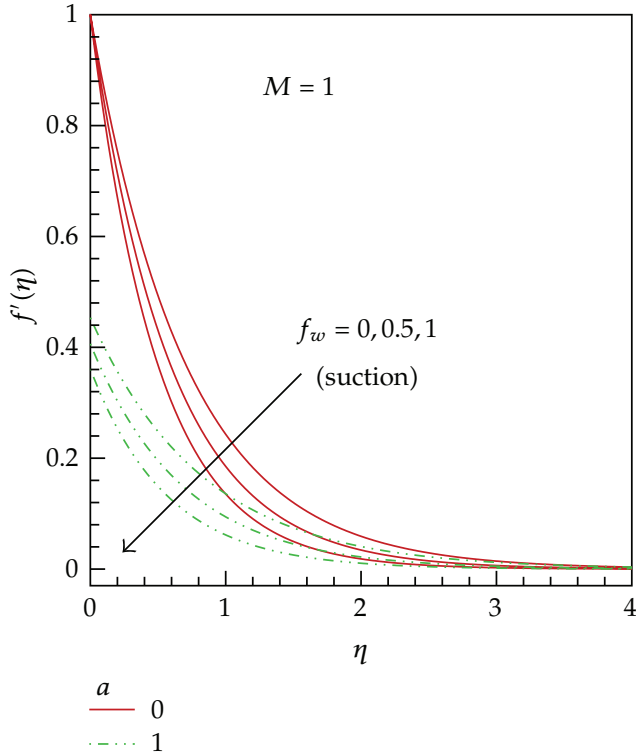

(a)

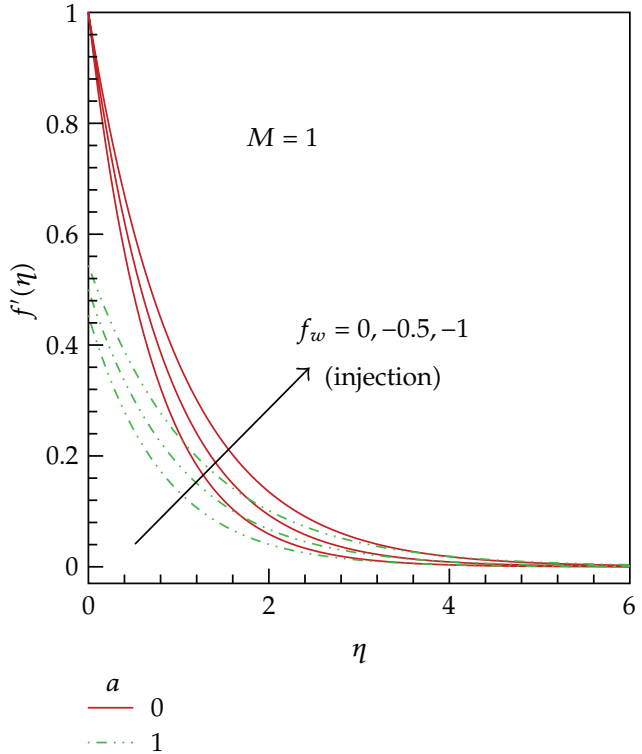

(b)

Figure 2: Effect of velocity slip parameter on dimensionless velocity for (a) suction and (b) injection.

to the buoyancy effect. On the other hand, an increase in the negative value of $Q$ will reduce thermal energy from the flow causing the velocity and the temperature distribution to drop.

\subsection{Skin Friction}

Figure 6 illustrates the variation of skin friction with suction/injection parameters for different values of magnetic (Figure 6(a)) and velocity slip parameters (Figure 6(b)). Figure 6(a) shows that an increase in suction parameter $f_{w}$ leads to a rise in the skin friction for both hydromagnetic and magnetohydrodynamic boundary layers, whereas an opposite behavior is found in case of injection. It is also found that the magnetic field leads to an increase in the skin friction factor. In the presence of the magnetic field, Figure 6(b), it is also observed that with an increase in suction parameter $f_{w}$, the skin friction factor increases for both slip and no-slip boundary conditions, whereas the opposite behavior was observed in case of injection. 


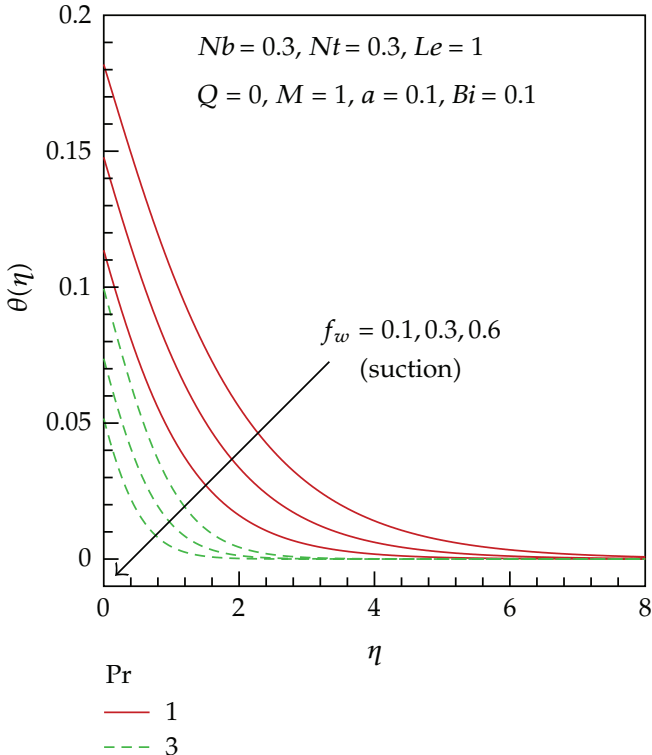

(a)

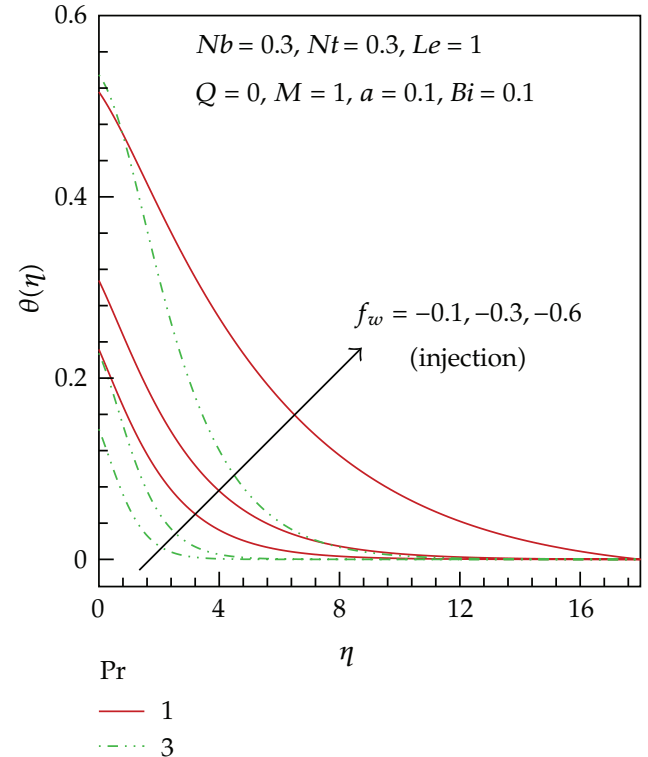

(b)

Figure 3: Effect of Prandtl number on dimensionless temperature for (a) suction and (b) injection.

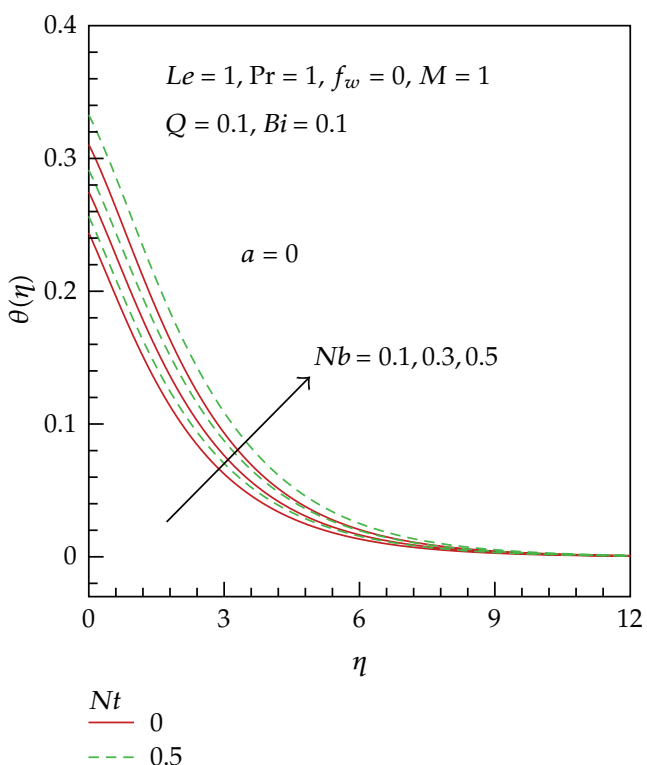

(a)

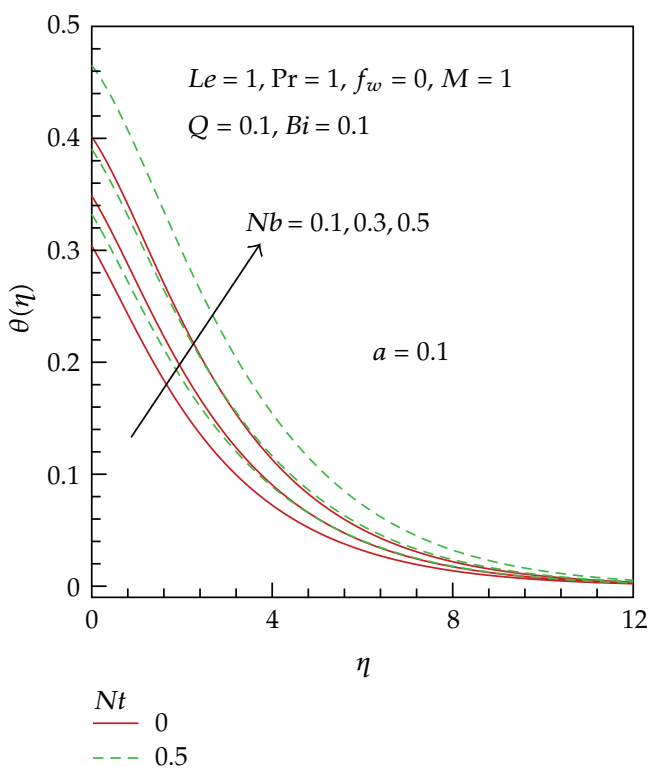

(b)

Figure 4: Effect of velocity slip and nanofluid parameters on dimensionless temperature. 


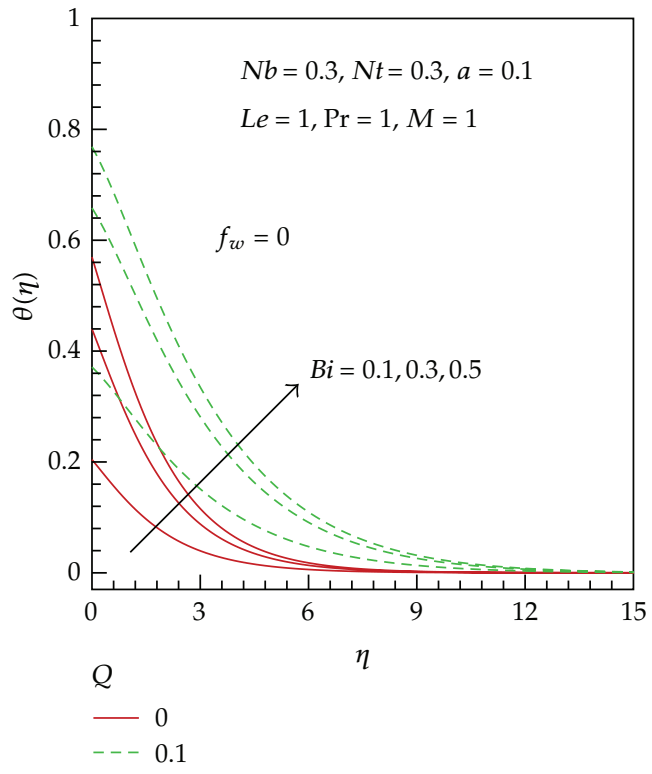

(a)

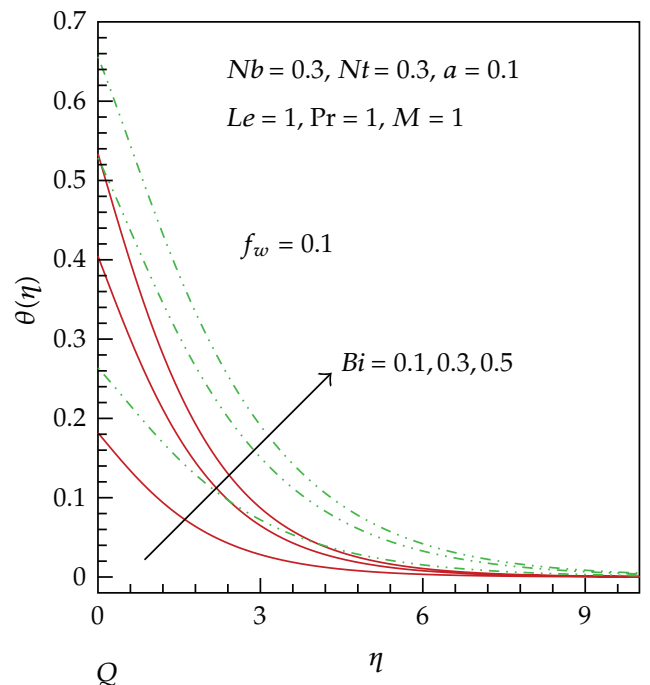

$-0$

(b)

Figure 5: Effect of Biot number and heat generation parameter on dimensionless temperature for (a) no suction/injection and (b) suction.

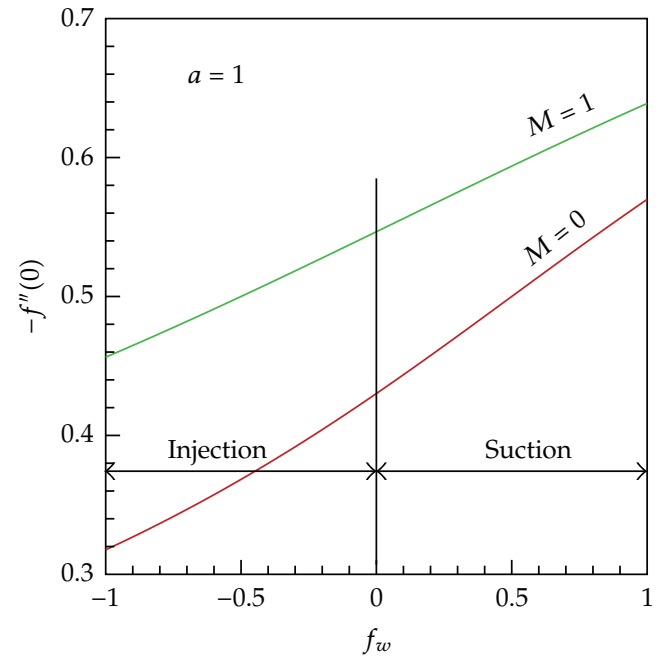

(a)

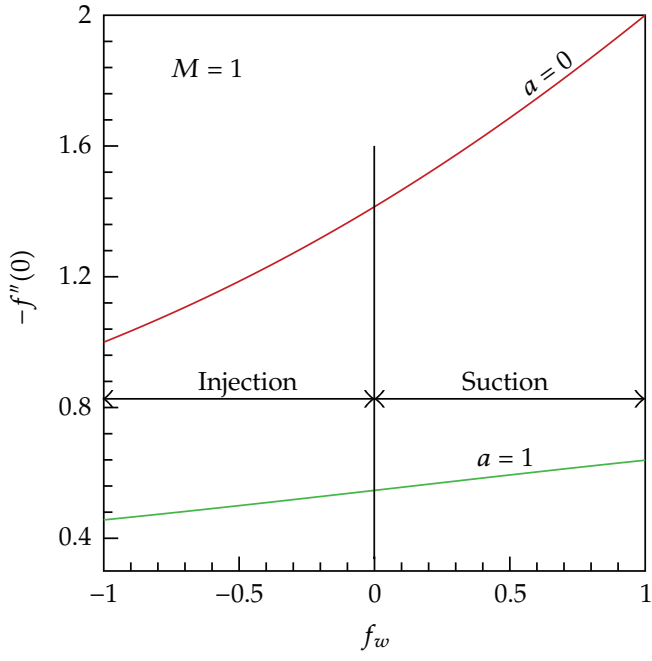

(b)

Figure 6: Variation of skin friction with suction/injection parameters for variable (a) magnetic and (b) velocity slip parameters.

\subsection{Heat Transfer Rate}

The variation of the dimensionless heat transfer rate with nanofluid and heat generation parameters is shown in Figure 7(a). It is observed that the dimensionless heat transfer rate decreases with an increase in heat generation and nanofluid parameters. This behavior was 


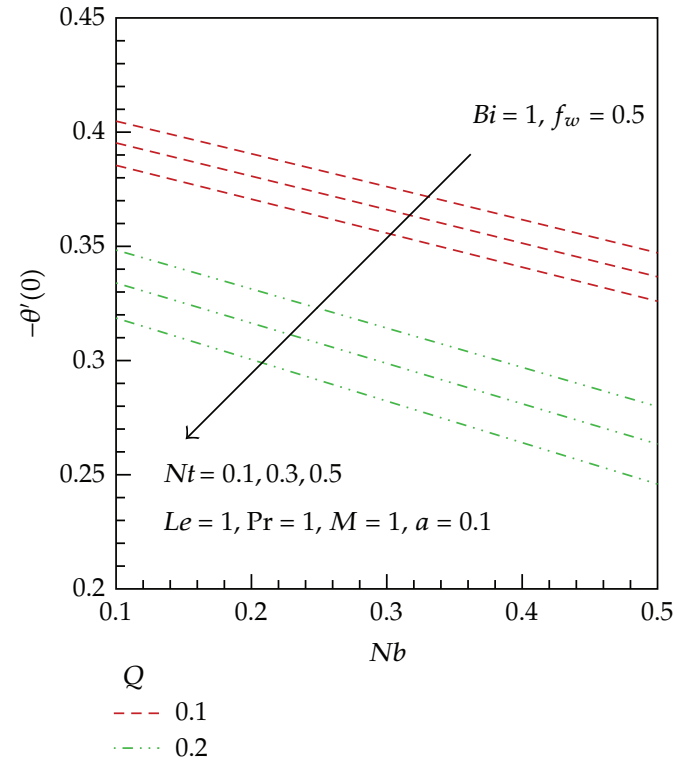

(a)

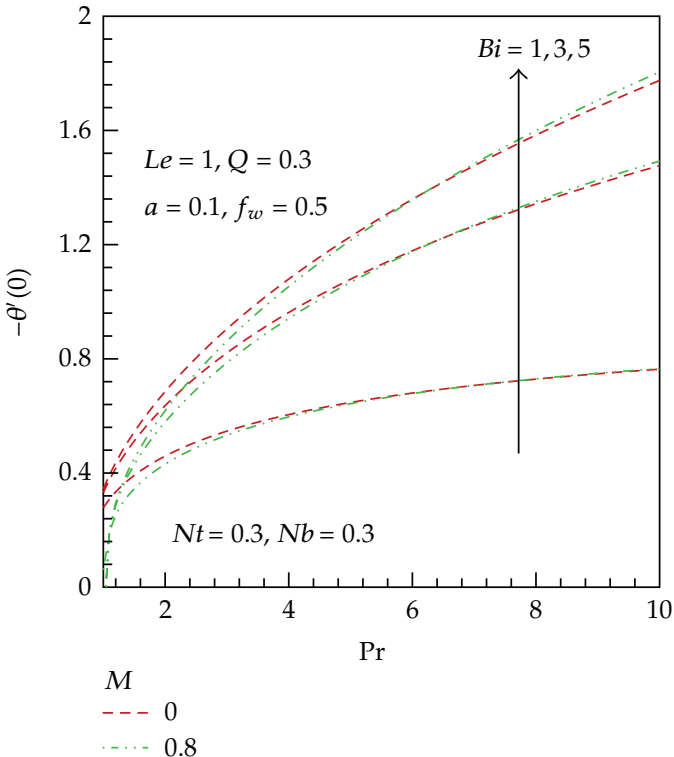

(b)

Figure 7: Effect of various parameters and dimensionless numbers on dimensionless heat transfer rates.

observed in case of suction. Figure 7(b) shows that as Prandtl and Biot numbers increase, the dimensionless heat transfer rate is increased for both the hydrodynamic $(M=0)$ and magnetohydrodynamic $(M \neq 0)$ thermal boundary layers. The dimensionless heat transfer rates are found to increase with Prandtl numbers. For small Prandtl numbers, no appreciable effect of magnetic parameter on the dimensionless heat transfer rate could be found.

\subsection{Nanoparticle Volume Fraction Rate}

The variation of dimensionless nanoparticle volume fraction rate with various flow controlling parameters is shown in Figure 8. From Figure 8(a), it is obvious that the nanoparticle volume fraction rate increases with the Brownian motion parameter and decreases with thermophoresis parameter for small values of heat generation $(Q=0.2)$. Opposite behavior is observed in case of higher heat generation $(Q=0.3)$. The nanoparticle volume fraction rate is increased with the Lewis number and suction parameter for both purely hydromagnetic and magnetohydrodynamic flows. No appreciable effect of magnetic field on the nanoparticle volume fraction rate could be found in case of suction (Figure 8(b)).

\section{Conclusions}

MHD boundary layer flow in a nanofluid over a convectively heated permeable linearly stretching sheet with heat generation is investigated numerically. The applications of momentum slip boundary condition instead of conventional no-slip boundary condition make our study more novel. Similarity representations of the governing equations are derived by scaling group of transformation instead of using the existing transformations. The following conclusions can be drawn. 


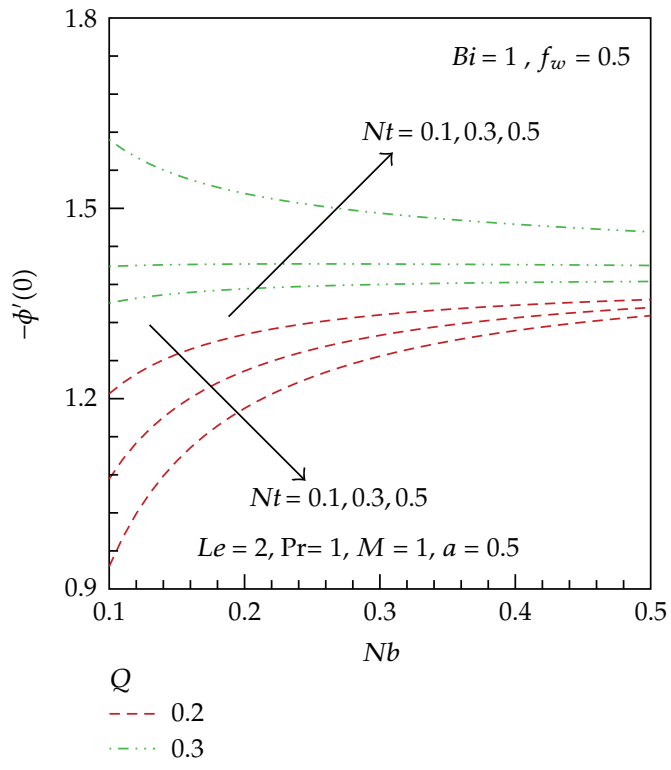

(a)

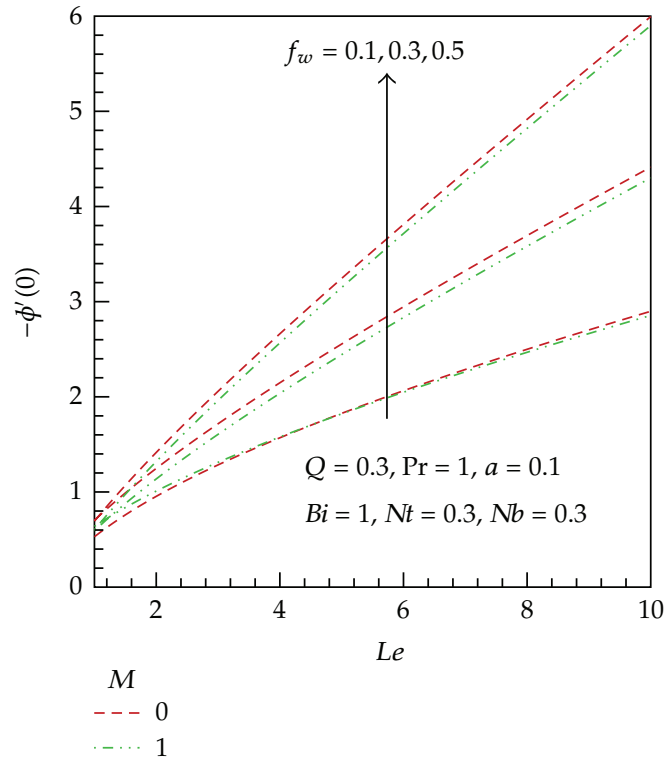

(b)

Figure 8: Effect of various parameters and dimensionless numbers on dimensionless mass transfer rates.

(i) The transport of momentum, energy, and nanoparticle volume fraction inside respective boundary layers depends upon six parameters, Brownian motion, thermophoresis, velocity slip, suction/injection, magnetic, and heat generation parameters, and three dimensionless numbers, Prandtl number Pr, Lewis number Le, and Biot number Bi.

(ii) For purely hydrodynamic boundary layer $(M=0)$, no-slip boundary conditions $(a=0)$ and impermeable plate $(f w=0)$ and in the absence of heat generation $(Q=0)$, our results are found in good agreement with Makinde and Aziz [25] and Khan and Pop [47].

(iii) Magnetic field reduces the velocity, heat transfer rate, and nanoparticle volume fraction rate.

(iv) With the increase in $a$, the velocity layer thickens but the temperature layer becomes thinner.

(v) Heat generation increases temperature.

(vi) Suction reduces whilst injection enhances the velocity boundary layer thickness for both magnetohydrodynamic and hydrodynamic boundary layer in the presence or absence of slip boundary condition. Similar behavior is found for temperature boundary layer.

(vii) Lewis number increases the nanoparticle volume fraction rate for both magnetohydrodynamic and hydrodynamic layers. Heat transfer rate is found to be increased with the Biot number. In the limiting case, when Bi tends to infinity, the results of heat transfer are comparable to the isothermal case. 
(viii) The skin friction factor increases with $M$ whilst it decreases with $a$. Suction increases skin friction factor for both magnetohydrodynamic and hydrodynamic layers with slip flow or without slip flow. Opposite behavior is noticed in case of injection.

(ix) The temperature of the fluid and thermal boundary layer thickness increases for both slip and no slip boundary conditions whereas the nanoparticle volume fraction decreases with increase of Brownian motion.

\section{References}

[1] M. Prodanovi, S. Ryoo, and A. R. Rahmani, "Effect of magnetic field on the motion of multiphase fluids containing paramagnetic nanoparticles in porous media," in SPE Improved Oil Recovery Symposium, Tulsa, Okla, USA, April 2010.

[2] K. Khanafer, K. Vafai, and M. Lightstone, "Buoyancy-driven heat transfer enhancement in a twodimensional enclosure utilizing nanofluids," International Journal of Heat and Mass Transfer, vol. 46, no. 19, pp. 3639-3653, 2003.

[3] S. Özerinç, S. Kakaç, and A. G. YazIcIoğlu, "Enhanced thermal conductivity of nanofluids: a state-ofthe-art review," Microfluidics and Nanofluidics, vol. 8, no. 2, pp. 145-170, 2010.

[4] R. Kandasamy, P. Loganathan, and P. P. Arasu, "Scaling group transformation for MHD boundarylayer flow of a nanofluid past a vertical stretching surface in the presence of suction/injection," Nuclear Engineering and Design, vol. 241, no. 6, pp. 2053-2059, 2011.

[5] R. P. E. Sharma, Nanostructuring Operations in Nanoscale Science and Engineering, McGraw-Hill, 2010.

[6] J. Buongiorno and W. Hu, "Nanofluid coolants for advanced nuclear power plants," in Proceedings of the International Congress on Advances in Nuclear Power Plants (ICAPP '05), Paper no. 5705, Seoul, Korea, May 2005.

[7] J. Buongiorno, "Convective transport in nanofluids," Journal of Heat Transfer, vol. 128, no. 3, pp. 240250, 2006.

[8] A. V. Kuznetsov and D. A. Nield, "Natural convective boundary-layer flow of a nanofluid past a vertical plate," International Journal of Thermal Sciences, vol. 49, no. 2, pp. 243-247, 2010.

[9] A. V. Kuznetsov and D. A. Nield, "Thermal instability in a porous medium layer saturated by a nanofluid: brinkman model," Transport in Porous Media, vol. 81, no. 3, pp. 409-422, 2010.

[10] A. V. Kuznetsov and D. A. Nield, "Effect of local thermal non-equilibrium on the onset of convection in a porous medium layer saturated by a nanofluid," Transport in Porous Media, vol. 83, no. 2, pp. 425-436, 2010.

[11] W. Duangthongsuk and S. Wongwises, "Effect of thermophysical properties models on the predicting of the convective heat transfer coefficient for low concentration nanofluid," International Communications in Heat and Mass Transfer, vol. 35, no. 10, pp. 1320-1326, 2008.

[12] E. Abu-Nada and H. F. Oztop, "Effects of inclination angle on natural convection in enclosures filled with Cu-water nanofluid," International Journal of Heat and Fluid Flow, vol. 30, no. 4, pp. 669-678, 2009.

[13] P. Rana and R. Bhargava, "Numerical study of heat transfer enhancement in mixed convection flow along a vertical plate with heat source/sink utilizing nanofluids," Communications in Nonlinear Science and Numerical Simulation, vol. 16, no. 11, pp. 4318-4334, 2011.

[14] A. J. Chamkha and A. M. Aly, "MHD free convection flow of a nanofluid past a vertical plate in the presence of heat generation or absorption effects," Chemical Engineering Communications, vol. 198, no. 3, pp. 425-441, 2011.

[15] R. S. R. O. Gorla and A. Chamkha, "Natural convective boundary layer flow over a horizontal plate embedded in a porous medium saturated with a nanofluid," Journal of Modern Physics, vol. 2, pp. 62-71, 2011.

[16] A. M. Rashad, M. A. EL-Hakiem, and M. M. M. Abdou, "Natural convection boundary layer of a non-Newtonian fluid about a permeable vertical cone embedded in a porous medium saturated with a nanofluid," Computers and Mathematics with Applications, vol. 62, pp. 3140-3151, 2011.

[17] L. J. Crane, "Flow past a stretching plate," Zeitschrift für angewandte Mathematik und Physik ZAMP, vol. 21, no. 4, pp. 645-647, 1970.

[18] S. Liao, "A new branch of solutions of boundary-layer flows over an impermeable stretched plate," International Journal of Heat and Mass Transfer, vol. 48, no. 12, pp. 2529-2539, 2005. 
[19] K. Vajravelu and J. R. Cannon, "Fluid flow over a nonlinearly stretching sheet," Applied Mathematics and Computation, vol. 181, no. 1, pp. 609-618, 2006.

[20] A. Raptis and C. Perdikis, "Viscous flow over a non-linearly stretching sheet in the presence of a chemical reaction and magnetic field," International Journal of Non-Linear Mechanics, vol. 41, no. 4, pp. 527$529,2006$.

[21] A. Y. Bakier, "Thermophoresis effects on heat and mass transfer in mhd flow over a vertical stretching surface with radiation," International Journal of Fluid Mechanics Research, vol. 36, no. 6, pp. 489-501, 2009.

[22] S. M. Abel, K. A. Kumar, and R. Ravikumara, "MHD flow, and heat transfer with effects of buoyancy, viscous and joules dissipation over a nonlinear vertical stretching porous sheet with partial slip," Engineering, vol. 3, pp. 285-291, 2011.

[23] A. Pantokratoras, "Study of MHD boundary layer flow over a heated stretching sheet with variable viscosity: a numerical reinvestigation," International Journal of Heat and Mass Transfer, vol. 51, no. 1-2, pp. 104-110, 2008.

[24] M. H. Yazdi, S. Abdullah, I. Hashim, and K. Sopian, "Slip MHD liquid flow and heat transfer over non-linear permeable stretching surface with chemical reaction," International Journal of Heat and Mass Transfer, vol. 54, no. 15-16, pp. 3214-3225, 2011.

[25] O. D. Makinde and A. Aziz, "Boundary layer flow of a nanofluid past a stretching sheet with a convective boundary condition," International Journal of Thermal Sciences, vol. 50, no. 7, pp. 1326-1332, 2011.

[26] A. Aziz, "A similarity solution for laminar thermal boundary layer over a flat plate with a convective surface boundary condition," Communications in Nonlinear Science and Numerical Simulation, vol. 14, no. 4, pp. 1064-1068, 2009.

[27] M. A. A. Hamad, M. Uddin, and A. I. M. Ismail, "Investigation of combined heat and masstransfer by Lie group analysis with variable diffusivity taking into account hydrodynamic slip and thermal convective boundary conditions," International Journal of Heat and Mass Transfer, vol. 55, pp. 1355-1362, 2012.

[28] S. Yao, T. Fang, and Y. Zhong, "Heat transfer of a generalized stretching/shrinking wall problem with convective boundary conditions," Communications in Nonlinear Science and Numerical Simulation, vol. 16, no. 2, pp. 752-760, 2011.

[29] N. A. Yacob, A. Ishak, and I. Pop, "Falkner-Skan problem for a static or moving wedge in nanofluids," International Journal of Thermal Sciences, vol. 50, no. 2, pp. 133-139, 2011.

[30] K. Bhattacharyya, S. Mukhopadhyay, and G. C. Layek, "Slip effects on boundary layer stagnationpoint flow and heat transfer towards a shrinking sheet," International Journal of Heat and Mass Transfer, vol. 54, no. 1-3, pp. 308-313, 2011.

[31] M. J. Martin and I. D. Boyd, "Falkner-Skan flow over a wedge with slip boundary conditions," Journal of Thermophysics and Heat Transfer, vol. 24, no. 2, pp. 263-270, 2010.

[32] G. M. Hak, "Flow physics in the MEMS handbook," in MEMS Handbook, M. Gad-el-Hak, Ed., chapter 4, CRC Press, Boca Raton, Fla, USA, 2002.

[33] M. T. Matthews and J. M. Hill, "Micro/nano thermal boundary layer equations with slip-creep-jump boundary conditions," IMA Journal of Applied Mathematics, vol. 72, no. 6, pp. 894-911, 2007.

[34] S. Mukhopadhyay, G. C. Layek, and S. A. Samad, "Study of MHD boundary layer flow over a heated stretching sheet with variable viscosity," International Journal of Heat and Mass Transfer, vol. 48, no. 21-22, pp. 4460-4466, 2005.

[35] M. Yurusoy, M. Pakdemirli, and O. F. Noyan, "Lie group analysis of creeping flow of a second grade fluid," International Journal of Non-Linear Mechanics, vol. 36, no. 6, pp. 955-960, 2001.

[36] D. Shang, Theory of Heat Transfer with Forced Convection Film Flows, chapter 3, Springer, New York, NY, USA, 2010.

[37] J. M. Hill, Differential Equations and Group Methods for Scientists and Engineers, CRC Press, Boca Raton, Fla, USA, 1992.

[38] M. Pakdemirli and M. Yurusoy, "Similarity transformations for partial differential equations," SIAM Review, vol. 40, no. 1, pp. 96-101, 1998.

[39] Y. Z. Boutros, M. B. Abd-El-Malek, N. A. Badran, and H. S. Hassan, "Lie-group method of solution for steady two-dimensional boundary-layer stagnation-point flow towards a heated stretching sheet placed in a porous medium," Meccanica, vol. 41, no. 6, pp. 681-691, 2006.

[40] A. Keçebaş and M. Yurusoy, "Similarity solutions of unsteady boundary layer equations of a special third grade fluid," International Journal of Engineering Science, vol. 44, no. 11-12, pp. 721-729, 2006. 
[41] A. M. Salem, "Temperature-dependent viscosity effects on non-darcy hydrodynamic free convection heat transfer from a vertical wedge in porous media," Chinese Physics Letters, vol. 27, no. 6, Article ID 064401, 2010.

[42] P. Puvi Arasu, P. Loganathan, R. Kandasamy, and I. Muhaimin, "Lie group analysis for thermaldiffusion and diffusion-thermo effects on free convective flow over a porous stretching surface with variable stream conditions in the presence of thermophoresis particle deposition," Nonlinear Analysis, vol. 5, no. 1, pp. 20-31, 2011.

[43] M. J. Uddin, W. A. Khan, and A. I. M. Ismail, "Free convective flow of a nanofluid from a convectively heated upward facing horizontal plate in porous media," Transport in Porous Media, vol. 92, no. 3, pp. 867-881, 2012.

[44] A. A. Avramenko, S. G. Kobzar, I. V. Shevchuk, A. V. Kuznetsov, and L. T. Iwanisov, “Symmetry of turbulent boundary-layer flows: investigation of different eddy viscosity models," Acta Mechanica, vol. 151, no. 1-2, pp. 1-14, 2001.

[45] S. Mukhopadhyay and G. C. Layek, "Effects of variable fluid viscosity on flow past a heated stretching sheet embedded in a porous medium in presence of heat source/sink," Meccanica, vol. 47, no. 4, pp. 863-876, 2011.

[46] T. Hayat, M. Qasim, and S. Mesloub, "MHD flow and heat transfer over permeable stretching sheet with slip conditions," International Journal for Numerical Methods in Fluids, vol. 66, no. 8, pp. 963-975, 2011.

[47] W. A. Khan and I. Pop, "Boundary-layer flow of a nanofluid past a stretching sheet," International Journal of Heat and Mass Transfer, vol. 53, no. 11-12, pp. 2477-2483, 2010.

[48] D. A. Nield and A. V. Kuznetsov, "The Cheng-Minkowycz problem for the double-diffusive natural convective boundary layer flow in a porous medium saturated by a nanofluid," International Journal of Heat and Mass Transfer, vol. 54, no. 1-3, pp. 374-378, 2011.

[49] T. Tapanidis, G. R. Tsagas, and H. P. Mazumdar, "Application of scaling group of Transformations to viscoelastic second-grade fluid flow," Nonlinear Functtional Analysis and Applications, vol. 8, no. 3, pp. 345-350, 2003.

[50] A. Aziz Uddin, M. J. Hamad, and M. A. A. Ismail, “MHD flow over an inclined radiating plate with the temperature dependent thermal conductivity, variable reactive index and heat generation," Heat Transfer Asian Research. In press.

[51] A. G. Hansen, Similarity Analysis of Boundary Layer Problems in Engineering, Prentice Hall, Englewood Cliffs, NJ, USA, 1964.

[52] W. F. Ames, Nonlinear Partial Differential Equations in Engineering, Academic Press, New York, NY, USA, 1972.

[53] A. Pantokratoras, "A common error made in investigation of boundary layer flows," Applied Mathematical Modelling, vol. 33, no. 1, pp. 413-422, 2009. 


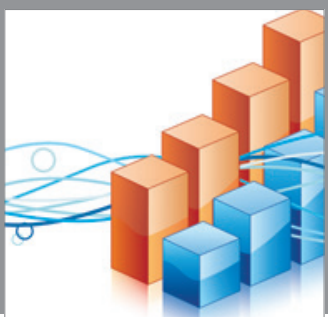

Advances in

Operations Research

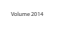

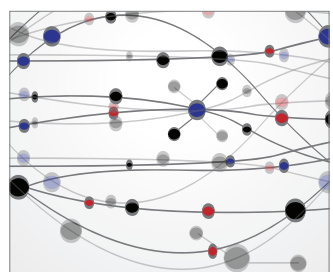

\section{The Scientific} World Journal
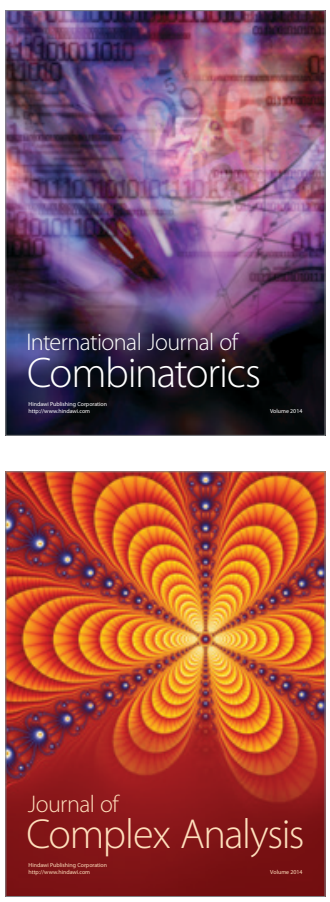

International Journal of

Mathematics and

Mathematical

Sciences
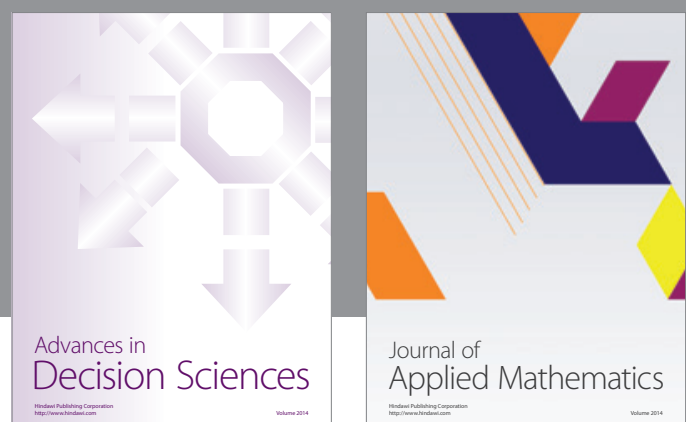

Journal of

Applied Mathematics
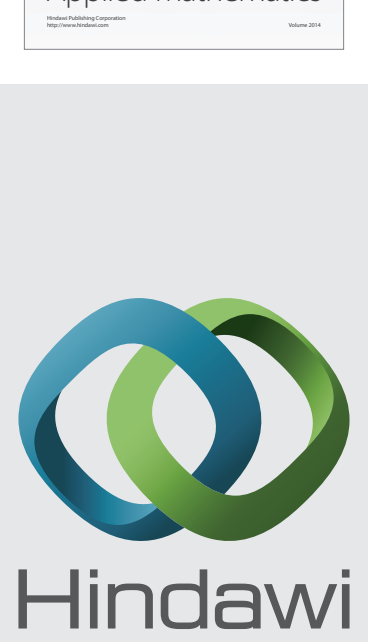

Submit your manuscripts at http://www.hindawi.com
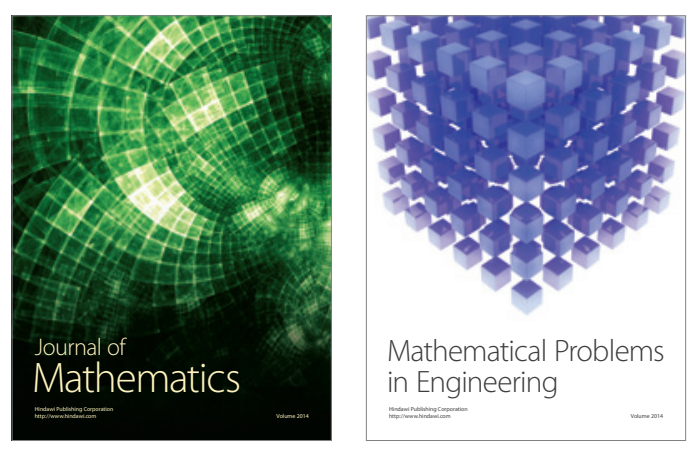

Mathematical Problems in Engineering
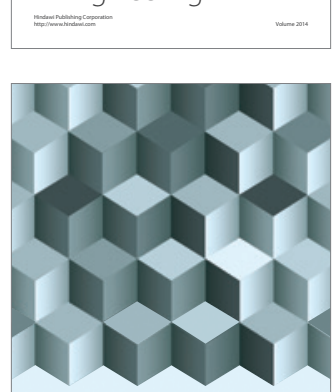

Journal of

Function Spaces
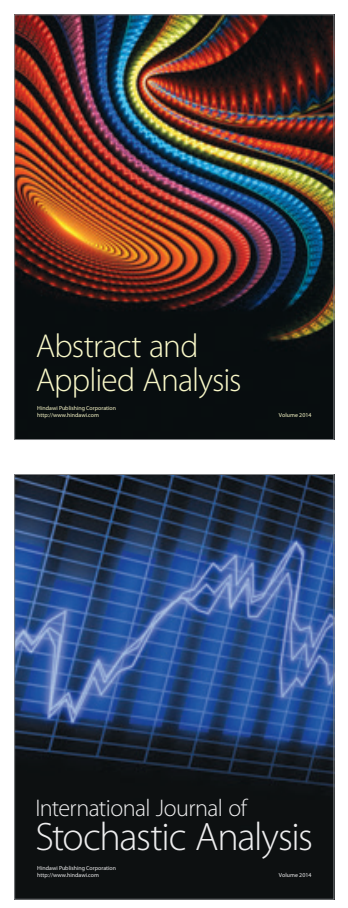

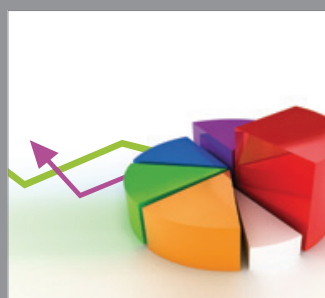

ournal of

Probability and Statistics

Promensencen
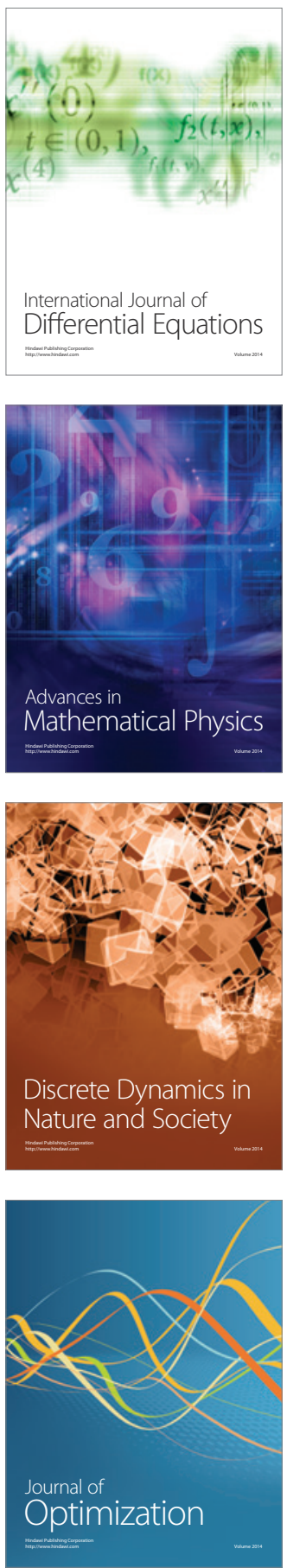